\title{
Quantifying the Lasting Effects of Resistance and Endurance Exercise Interventions on Mental Wellbeing and Physical Fitness in Women Receiving Adjuvant Treatment for Breast Cancer Compared to Adjuvant Therapy Alone: A Contemporary Systematic Review and Meta-Analysis
}

Jonathon Mok

Loughborough University

Marie-Juliet Brown

Loughborough University

Elizabeth Akam

Loughborough University

Mhairi Morris ( $\square$ m.a.morris@lboro.ac.uk)

Loughborough University

Research Article

Keywords: interventions, resistance, adjuvant, meta, endurance, breast, cancer

Posted Date: July 16th, 2021

DOI: https://doi.org/10.21203/rs.3.rs-701571/v1

License: (c) (i) This work is licensed under a Creative Commons Attribution 4.0 International License. Read Full License 


\section{Abstract}

Introduction: Breast cancer is a persisting global burden for health services with cases and deaths projected to rise in future years. Surgery complemented by adjuvant therapy is commonly used to treat breast cancer, however comes with detrimental side effects to physical fitness and mental wellbeing.

Aim: The aim of this systematic review and meta-analysis is to determine whether resistance and endurance interventions performed during adjuvant treatment can lastingly ameliorate these side effects.

Methods: A systematic literature search was performed in various electronic databases. Papers were assessed for bias and grouped based on intervention design. RStudio was used to perform the metaanalyses for each group using the 'meta' package. Publication bias and power analyses were also conducted. These methods conform to PRISMA guidelines.

Results: Combined resistance and endurance interventions elicited significant long-lasting improvements in global fatigue and were beneficial to the remaining side effects. Individually, resistance and endurance interventions non-significantly improved these side effects. Resistance interventions elicited higher benefits overall.

Conclusion: Exercise interventions have lasting clinical benefits in ameliorating adjuvant therapy side effects, which negatively impact physical fitness and mental wellbeing. These interventions are of clinical value to enhance adherence rates and avoid comorbidities such as sarcopenia, thus improving disease prognosis.

\section{Key Points}

This systematic review and meta-analysis is the first of its kind to investigate whether resistance and endurance exercise interventions can have a lasting impact on the reduction of side effects from adjuvant chemotherapy in breast cancer patients. The results presented herein demonstrate that both combined (resistance plus endurance) and individual exercise interventions (resistance alone or endurance alone) are of lasting clinical benefit to reducing the negative impact of chemotherapy on physical fitness and mental wellbeing.

\section{Introduction}

In 2020, there were approximately 19.3 million new cancer cases globally, of which, female breast cancer was the highest contributor at $11.7 \%$ of the total. As a result of these new cases, there were 10 million deaths attributed to cancer: female breast cancer constituted $6.9 \%$ of these deaths $(684,996$ deaths) [1]. The number of new breast cancer cases and mortality rates are only projected to rise in future years, thus female breast cancer represents a significant burden on female health and health services. 
Currently, multiple treatment options exist to treat breast cancer. These primarily involve surgery to remove the tumour, usually a mastectomy or breast conserving surgery, which are followed up by adjuvant therapy such as chemotherapy, radiotherapy, hormone therapy or forms of targeted therapy. This ensures the tumour is removed and the risk of relapse is reduced due to a decreased risk of metastasis that results from adjuvant therapy [2]. In 2019, breast conserving surgery followed up with adjuvant radiotherapy was the most common form of treatment for early breast cancer in stages I and II in American female breast cancer patients (49\%) [3]. For more severe breast cancers in stages III and IV, chemotherapy and hormonal therapy were the most common form of treatments in American female patients $-56 \%$ and $71 \%$ of all cases were treated with these, respectively [3].

While adjuvant therapy has shown much success in recent years by extending overall survival and disease-free survival in breast cancer patients [4], adjuvant treatment also causes various unwanted lifechanging side effects. Common side effects include disturbances to mental wellbeing manifested in depression and fatigue, leading to an overall decreased quality of life (QOL) [5]. Other well-documented side effects include declines in physical fitness, manifested in reduced muscular strength and endurance following treatment [6]. These may decrease physical capacity and therefore daily physical functioning, which may also contribute to decreased adherence to treatment, ultimately decreasing the efficacy of adjuvant treatment. These side effects are therefore important to manage and enhance adherence rates boosting the efficacy of treatment options and therefore disease prognosis.

Generally, exercise is well characterised to reduce the risk of developing breast cancer and to reduce the mortality rates linked to breast cancers. McTiernan et al. [7] show that the risk of developing breast cancer is reduced by up to $18 \%$ when exercise is performed regularly. Alongside this, Palesh et al. [8] demonstrated that an hour a day of moderate physical activity decreases the mortality of advanced breast cancers by $23 \%$. Specifically, resistance and endurance exercise designs are typically used in the array of studies investigating the effects of exercise on breast cancer survival and risk. Resistance exercise is defined as using resistance in the form of weights or resistance bands to elicit muscular hypertrophy [9] whereas endurance exercise is the continuous activation of skeletal muscle groups over a prolonged period of time to improve aerobic capacity [10].

While many reviews have characterised the beneficial effects of exercise on breast cancer survival and mortality, no reviews to date have quantified the effects of resistance and endurance interventions to ameliorate the detrimental side effects impacting physical fitness and mental wellbeing that come with adjuvant therapy in order to avoid further pathology and improve daily functioning which may boost the efficacy of these treatments. In addition, whether the beneficial effects of exercise to ameliorate these side effects are lasting is yet to be elucidated.

Therefore, the aims of this meta-analysis and systematic review are:

1. 1. To quantify the lasting effects of combined resistance and endurance interventions on physical fitness and mental wellbeing in female breast cancer patients ( $\geq 18$ years old) undergoing adjuvant 
therapy by measuring the following factors: cardiorespiratory fitness, depression, fatigue, muscular endurance, muscular strength, quality of life $(\mathrm{QOL})$ and social functioning.

2. 2. To quantify the lasting effects of interventions consisting of only resistance or only endurance exercise on these factors and to elucidate which type of exercise is more effective (by comparison) in improving mental wellbeing and physical fitness in patients undergoing adjuvant therapy.

\section{Materials And Methods \\ 2.1 Search Method}

This systematic review and meta-analysis conforms to the Preferred Reporting Items for Systematic Reviews and Meta-Analyses (PRISMA) guidelines [11]. To obtain papers for this meta-analysis, a comprehensive systematic literature search was conducted in the following electronic databases: PubMed, BioMed Central (BMC), Scopus, Web of Science Core collection, Cochrane Library and Ovid with the last search being conducted in December 2020. Search terms to obtain these papers used the Boolean operator "AND" to narrow the results returned and terms started off broadly such as "exercise" AND "cancer" to identify the knowledge gap in the field of exercise oncology. These terms progressively became more specific to pinpoint required papers to answer the knowledge gap. Specific search terms included "endurance" AND "resistance exercise" "on breast cancer". A full list of search terms used to conduct the literature search are listed in Table 1 in the appendix. The inclusion criteria to select these papers is as follows: study was a published randomised controlled trial; a published clinical trial with a complete dataset; used human participants; contained endurance/aerobic or resistance exercise interventions lasting a minimum of 20 minutes per session; investigated at least one of the outcome measures required; was written in English; was published from 2010-2020; is exclusive to breast cancer; is a $4+$ star paper (OVID); and is open access or accessible through the Loughborough University Library.

\subsection{Outcome Measures}

Outcome measures obtained from each study that met the inclusion criteria were cardiorespiratory fitness, depression, global fatigue, muscular endurance, muscular strength, quality of life and social functioning. Cancer related fatigue was used as a substitute where global fatigue was not measured. Cardiorespiratory fitness, muscular endurance and muscular strength constitute the umbrella term "physical fitness", and depression, global fatigue, quality of life and social functioning constitute the umbrella term "mental wellbeing". These were all continuous outcomes.

\subsection{Data Extraction and Risk of Bias}

Data (means, standard deviations and numbers of participants) concerning the above outcome measures were extracted from baseline and from the last available time points in each study that reached the inclusion criteria from both the exercise intervention and control conditions. If the data was not immediately available, the corresponding authors were contacted directly via email requesting the relevant data. If the authors were unable to reply, their papers were excluded from the meta-analyses. 
Each paper reaching the inclusion criteria was assessed for risk of bias using the National Toxicology Program's Office of Health Assessment and Translation (OHAT) Risk of Bias rating tool [12]. The questions used for assessment are as follows: 1) Was administered dose or exposure level adequately randomised? 2) Was allocation to study groups adequately concealed? 3) Did selection of study participants result in appropriate comparison groups? 4) Did the study design or analysis account for important confounding and modifying variables? 5) Were the research personnel and human subjects blinded to the study group during the study? 6) Were outcome data complete without attrition or exclusion from analysis? 7) Can we be confident in the exposure characterization? 8) Can we be confident in the outcome assessment? 9) Were all measured outcomes reported? 10) Were there no other potential threats to internal validity? Risk of bias analysis was also carried out by another researcher using the OHAT tool to avoid bias which may arise from singular opinion. To gain an overall rating for each study, a numerical system was deployed which takes into account each question equally. In this, the rating "Definitely low" risk equated to +4 points, "Probably low" risk equated to +2 , "Definitely High" risk equated to -4 and "Probably low" risk equated to -2 points. An average of these scores was then taken for each study across the 10 domains. If the average score was between +2 to +4 the rating was "Definitely low" risk, +0.1 to +1.9 was "Probably low" risk, -2 to -4 was "Definitely High" risk and 0 to -1.9 was "Probably high" risk. The rating "NR" was excluded from this average calculation. Disparities in the rating of the studies were resolved by discussion and a consensus was reached.

\subsection{Data Handling and Statistical Analysis}

Once all the necessary data was extracted, papers were sorted into 4 groups by intervention design to answer the aims of this study. The first group consisted of papers with interventions that used both resistance and endurance exercise. The second group consisted of papers with an exercise intervention consisting of solely resistance exercise while the third group consisted of papers with endurance interventions only. A fourth group was established which consisted of papers that compared resistance exercise to endurance exercise by using interventions consisting of both resistance and endurance as the intervention condition and interventions with just endurance as the control. Within these groups, the papers were grouped again by which of the outcome measures they investigated. Using these categories, a meta-analysis was carried out for each factor in each of the 4 groups. This allowed the investigation of the effects of having both resistance and endurance exercise on the outcome measures, the effects of having just resistance or endurance exercise and the effects of adding resistance to endurance exercise on the outcome measures (to further quantify which design was more effective) respectively. This process is graphically presented in Fig. 1 .

To conduct the meta-analyses, RStudio was used. Within RStudio, the 'meta' package was loaded enabling the 'metacont' function to be used to calculate effect sizes and 95\% confidence intervals of each study. The summary measure used was Standardised Mean Difference (SMD) with Hedges' g correction with the Q-profile method being used to calculate confidence intervals. Prediction intervals were also calculated for each factor where available, with both the fixed effects model and random effects model also being calculated. The percentage of variability in effect sizes across studies $\left(I^{2}\right)$ was used to 
determine which effects model was reported. When using the random effects model the Hartung-Knapp adjustment was applied to minimise Type 1 error rates [13]. The Inverse Variance method was used in each meta-analysis to calculate the weight/contribution of each study to the overall effect size displayed. To quantify between-study variance $\left(\mathrm{Tau}^{2}\right)$, Restricted Maximum-Likelihood (REML) was used due to being low in bias and yielding low Mean Squared Errors (MSE) of $\mathrm{Tau}^{2}$ for the number of studies and sample sizes used in these meta-analyses [14]. In the event that REML could not converge on a Tau ${ }^{2}$ estimate, the Sidik-Jonkman-type estimator (SJ) was used as an alternative due to having low bias estimates of $\mathrm{Tau}^{2}$. To summarise this data, forest plots were created for each variable using the 'forest.meta' function within the 'meta' package.

To investigate publication bias, a funnel plot was constructed using the 'meta' package and 'funnel' function, encompassing all of the studies included in the meta-analyses. To statistically quantify this, the Egger's test of intercept was calculated using the 'dmetar' package enabling the use of the function 'eggers.test'. Assessing for publication bias ensures the true effect sizes calculated are representative and not inflated due to studies finding small effect sizes not being published/included.

For each meta-analysis conducted, power analysis was carried out to quantify whether there was sufficient power to detect a statistically significant effect size where one exists. This was performed using the 'power.analysis' function as part of the 'dmetar' package [15]. Where random-effects models were reported, heterogeneity levels $\left(\mathrm{I}^{2}\right)$ for usage in the power calculation were defined using the following categories: $25 \%$ = Low, $50 \%$ = Moderate and $75 \%$ = High [16] .

\section{Results}

\subsection{Study Selection}

Once the gap in research was identified, 9488 papers were first obtained using the search terms "endurance" AND "resistance exercise" "on breast cancer". These papers were screened to check if they met the inclusion criteria stated previously and if the abstract, intervention design and outcome measures were relevant to these meta-analyses. Of these papers, 9372 were removed. 41 duplicate papers were also removed. This left 75 full-text papers which were assessed for eligibility based on content. This resulted in a further 57 papers being excluded leaving 18 papers to be used in these meta-analyses. This process is shown in Fig. 2.

\subsection{Study sorting}

The 18 selected papers were sorted into their respective groups using the method described previously, to perform the meta-analyses required. This is shown in Table 2 (appendix).

\subsection{Risk of Bias}

Of the 18 papers selected, 16 were shown to be "definitely low" in risk when considering all 10 questions. 1 paper was shown to be "probably high" in risk while the other paper was "probably low" in risk (Fig. 3). 


\subsection{Publication Bias}

The studies used for each factor and group exhibited no publication bias. This was shown by funnel plot symmetry and was statistically confirmed by Egger's test being non-significant $(P=0.176)$. This is shown in Fig. 4.

\subsection{Meta-Analyses}

\subsubsection{Combined Interventions Comprising Both Resistance and Endurance Exercise: Cardiorespiratory Fitness}

Of the 18 studies selected, six were used to investigate the effects of combined interventions consisting of both resistance and endurance exercise on cardiorespiratory fitness in women undergoing adjuvant therapy. Five out of six studies showed a positive effect size while one study showed a low negative effect size. Collectively, using the random effects model due to high heterogeneity, the overall effect size was non-significant low positive $\left(S M D=0.33,95 \% \mathrm{Cl}=[-0.09 ; 0.76], I^{\wedge} 2=71 \%, \mathrm{P}=0.09\right)$. Power analysis revealed this meta-analysis to have an optimal level of power of $80.23 \%$. Prediction intervals suggest future studies will favour a positive effect size (Fig. 5a).

\subsubsection{Combined Interventions Comprising Both Resistance and Endurance Exercise: Depression}

Two studies were used to quantify the effects of combined exercise interventions on depression. Out of the two studies, one showed a large negative effect size while the other showed a low positive. Reporting the random effects model, the overall effect size was found to be non-significant low negative (SMD = $\left.-0.42,95 \% \mathrm{Cl}=[-7.75 ; 6.91], I^{\wedge} 2=79 \%, \mathrm{P}=0.60\right)$. Power analysis shows this meta-analysis to have low power to detect a statistically significant effect size where one exists at $50.35 \%$. Due to only being able to use two studies, prediction intervals could not be created. This is shown in Fig. 5b.

\subsubsection{Combined Interventions Comprising Both Resistance and Endurance Exercise: Global Fatigue}

Five studies were used to investigate the effects of combined exercise interventions on global fatigue. All but one study showed a negative effect size, with the remaining one showing no effect. Collectively, a significant negative effect size was found when reporting the fixed effects model due to a lack of heterogeneity found by both REML and $S J\left(S M D=-0.26,95 \% \mathrm{Cl}=[-0.46 ;-0.07], I^{\wedge} 2=0 \%, \mathrm{P}=0.008\right)$. This was however accompanied by less-than-optimal statistical power (74.55\%). Prediction intervals suggest this will be also found in future studies (Fig. 5c)

\subsubsection{Combined Interventions Comprising Both Resistance and Endurance Exercise: Muscular Endurance}


No studies could be found with the desired inclusion criteria that investigated the effects of combined interventions on muscular endurance.

\subsubsection{Combined Interventions Comprising Both Resistance and Endurance Exercise: Muscular Strength}

Four of the five studies used to investigate the effects of combined resistance and endurance interventions on muscular strength found positive effect sizes. Collectively, using the random effects model these studies showed a small non-significant positive effect size $(\mathrm{SMD}=0.47,95 \% \mathrm{Cl}=[-0.46$; 1.40], $\left.I^{\wedge} 2=87 \%, P=0.235\right)$. Prediction intervals also favour a positive effect size in future studies. Optimal power was also achieved in this meta-analysis (91.86\%). This is demonstrated in Fig. 5d.

\subsubsection{Combined Interventions Comprising Both Resistance and Endurance Exercise: Quality of Life}

Overall, a non-significant low positive effect size was observed with low heterogeneity using the random effects model but with low statistical power of $27.98 \%\left(S M D=0.18,95 \% \mathrm{Cl}=[-0.27 ; 0.63], \mathrm{I}^{\wedge} 2=31 \%, \mathrm{P}=\right.$ 0.295). Prediction intervals also support this indicating a positive effect size will likely to be found in future studies (Fig. 5e).

\subsubsection{Combined Interventions Comprising Both Resistance and Endurance Exercise: Social Functioning}

Only one study was available to quantify the effects of combined resistance and endurance interventions on social functioning. Dong et al. [18] showed a non-significant positive effect size $(\mathrm{SMD}=0.26,95 \% \mathrm{Cl}=$ [-0.33; 0.86], $P=0.39)$. Power analysis and prediction intervals could not be carried out.

\subsubsection{Interventions Comprising Solely Resistance Exercise: Cardiorespiratory Fitness}

Only one study could be found with the desired inclusion criteria that investigated the lasting effects of solely resistance interventions on cardiorespiratory fitness during adjuvant treatment. Bolam et al. [25] showed a non-significant low positive effect size favouring the resistance intervention (SMD = 0.21, 95\% $\mathrm{Cl}=[-0.17 ; 0.59], \mathrm{P}=0.283)$. The direction of future studies however is unclear due to not being able to generate prediction intervals. In addition, power analysis could not be carried out.

\subsubsection{Interventions Comprising Solely Resistance Exercise: Depression}

Two studies were found that matched the inclusion criteria were used to investigate the long-lasting effects of resistance exercise on depression in female breast cancer patients undergoing adjuvant therapy. Reporting the fixed effects model, collectively they showed a non-significant small negative effect size $\left(S M D=-0.02,95 \% \mathrm{Cl}=[-0.28 ; 0.24], I^{\wedge} 2=0 \%, P=0.895\right)$. This meta-analysis however had low power at $5.26 \%$. This is shown in Fig. 6 a. 


\subsubsection{Interventions Comprising Solely Resistance Exercise: Global Fatigue}

Five studies were found to be eligible using the inclusion criteria to investigate the effects of resistance interventions on global fatigue. All studies displayed negative effect sizes giving a non-significant negative overall effect size using the random effects model $\left(S M D=-0.28,95 \% \mathrm{Cl}=[-0.56 ; 0.01], \Lambda^{\wedge} 2=14 \%\right.$, $P=0.055)$. Prediction intervals also favour this. Power analysis showed sub-optimal power to detect significance where it exists using these studies at $74.05 \%$ (Fig. 6b).

\subsubsection{Interventions Comprising Solely Resistance Exercise: Muscular Endurance}

Two studies were suitable to quantify the enduring effects of resistance interventions on muscular endurance during adjuvant therapy for breast cancer. Collectively, reporting the random effects model, a large non-significant positive effect size was observed, favouring the intervention, with high power at $96 \%$ $\left(S M D=1.01,95 \% \mathrm{Cl}=[-4.30 ; 6.32], I^{\wedge} 2=74 \%, P=0.25\right)$. However, due to the lack of studies to investigate this relationship, prediction intervals could not be performed. This is demonstrated in Fig. 6c.

\subsubsection{Interventions Comprising Solely Resistance Exercise: Muscular Strength}

Four studies that matched the inclusion criteria were used to quantify the effects of resistance interventions on muscular strength during adjuvant treatment. All four studies showed positive effect sizes favouring the intervention and gave a cumulative moderate positive effect size using the random effects model $\left(S M D=0.64,95 \% \mathrm{Cl}=[-0.25 ; 1.53], \wedge^{\wedge} 2=76 \%, P=0.11\right)$. Prediction intervals suggest future studies will also obtain similar findings and power analysis shows optimal power to detect a significant effect size where one exists at $98.62 \%$. This was however not statistically significant (Fig. 6d).

\subsubsection{Interventions Comprising Solely Resistance Exercise: Quality of Life}

Five studies were found to be eligible for this meta-analysis. Four studies exhibited positive effect sizes with the other was negative. Together reporting the random effects model, they gave a low positive nonsignificant effect size $\left(S M D=0.19,95 \% \mathrm{Cl}=[-0.29 ; 0.68], I^{\wedge} 2=62 \%, \mathrm{P}=0.33\right)$. Prediction intervals also reflect this. Power analysis showed there to be poor power to detect a significant effect size at $33.64 \%$ (Fig. 6e).

\subsubsection{Interventions Comprising Solely Resistance Exercise: Social Functioning}

Using the random effects model, cumulatively, three studies showed a low positive effect size when investigating the effects of resistance interventions on social functioning $(S M D=0.3095 \% \mathrm{Cl}=[-0.87$; 
1.46], $\wedge^{\wedge} 2=73 \%, P=0.39$ ). Power analysis showed poor power (38.68\%), with prediction intervals being very broad so displayed no clear direction. This is shown in Fig. $6 \mathrm{f}$.

\subsubsection{Interventions Comprising Solely Endurance Exercise: Cardiorespiratory Fitness}

Two studies were used to quantify the effects of endurance interventions on cardiorespiratory fitness. Both of these showed positive effect sizes and together gave a large positive effect size when reporting the random effects model with optimal statistical power at 99.71\% (SMD $=1.38,95 \% \mathrm{Cl}=[-17.09 ; 19.84]$, ${ }^{\wedge} 2=90 \%, P=0.52$ ). Since only two studies were used, the $95 \% \mathrm{Cl}$ was very large and prediction intervals were not able to be synthesised (Fig. 7a).

\subsubsection{Interventions Comprising Solely Endurance Exercise: Depression}

No studies were found to be eligible to investigate the effects of endurance interventions on depression.

\subsubsection{Interventions Comprising Solely Endurance Exercise: Global Fatigue}

Three studies were used to quantify the impact of endurance interventions on global fatigue during adjuvant therapy and collectively using the random effects model, they showed a non-significant low negative effect size $\left(S M D=-0.10,95 \% \mathrm{Cl}=[-1.14 ; 0.93],\left.\right|^{\wedge} 2=50 \%, P=0.71\right)$. This finding was however non-significant with low statistical power $(7.82 \%)$. Prediction intervals show no definitive future direction (Fig. 7b).

\subsubsection{Interventions Comprising Solely Endurance Exercise: Muscular Endurance}

Only one study was available to be used to investigate the effects of endurance interventions of muscular endurance. Schmidt et al. [28] gave a non-significant positive effect size (SMD $=0.37,95 \% \mathrm{Cl}=[-0.22$; $0.96], P=0.22)$. Prediction intervals and power analysis could not be carried out.

\subsubsection{Interventions Comprising Solely Endurance Exercise: Muscular Strength}

Two studies were used for this meta-analysis, both displaying negative effect sizes. Using the fixed effects model, the overall effect size was non-significant negative (SMD $=-0.10,95 \% \mathrm{Cl}=[-0.43 ; 0.22],{ }^{\wedge} 2$ $=0 \%, P=0.22)$. There was however low statistical power $(9.33 \%)$ and no prediction intervals could be synthesised. This is shown in Fig. 7c.

\subsubsection{Interventions Comprising Solely Endurance Exercise: Quality of Life}


Collectively, the three studies selected to investigate the effects of endurance interventions on QOL during adjuvant treatment showed a non-significant positive effect size when reporting the random effects model $\left(S M D=0.20,95 \% \mathrm{Cl}=[-0.69 ; 1.10], I^{\wedge} 2=28 \%, \mathrm{P}=0.43\right)$. There was however poor statistical power in this meta-analysis (19.63\%). Prediction intervals showed no clear direction (Fig. 7d).

\subsubsection{Interventions Comprising Solely Endurance Exercise: Social Functioning}

Two studies were used to investigate the impact of endurance interventions on social functioning. Both of these showed positive effect sizes and together gave a non-significant positive effect size when reporting the fixed effects model $\left(S M D=0.18,95 \% \mathrm{Cl}=[-0.14 ; 0.51], \wedge^{\wedge} 2=0 \%, P=0.27\right)$. This finding was non-significant with low statistical power (19.4\%). Prediction intervals could not be generated (Fig. 7e).

\subsection{Resistance and Endurance Interventions vs Endurance Interventions Alone}

To further explore which of the two interventions were better alone, two studies were used which both contained a 'COMB' (both resistance and endurance interventions) and a 'STAN' (endurance only) condition. The COMB was used as the exercise condition while STAN was used as the control. From the 7 outcome measures, 5 were available to measure. Overall using the random effects model, the metaanalysis gave a non-significant moderate positive effect size with optimal power $(99.9 \%)(S M D=0.56$, $\left.95 \% \mathrm{Cl}=[-0.72 ; 1.85],\left.\right|^{\wedge} 2=97 \%, \mathrm{P}=0.29\right)$. Prediction intervals confirmed this for future studies (Fig. 8).

\section{Discussion}

To our knowledge, this systematic review and meta-analysis is the first to date characterizing the lasting effects of combined exercise interventions on physical fitness and mental wellbeing during adjuvant therapy using the factors investigated herein.

These meta-analyses show interventions consisting of both resistance and endurance exercise elicit significant long-lasting improvements in global fatigue $\left(S M D=-0.26,95 \% \mathrm{Cl}=[-0.46 ;-0.07], \iota^{\wedge} 2=0 \%, \mathrm{P}=\right.$ 0.008). This finding is supported by Carayol et al. [35] who also finds exercise interventions consisting of resistance, aerobic, and yoga exercise significantly improve fatigue in breast cancer patients receiving adjuvant therapy $(P<0.0001)$. This is of importance because high levels of fatigue during adjuvant treatment have been significantly linked to decreased adherence to treatment. This is demonstrated by Kidwell et al. [36] who show patients that were feeling tired/fatigued had significantly decreased adherence to aromatase inhibitor adjuvant therapy compared to patients without this symptom (OR = 1.76). This is also supported by Ruddy et al. [37] who show cyclophosphamide-methotrexate-5fluorouracil (CMF) treatment attrition rates were significantly linked to patient fatigue $(P=0.025)$. Therefore, this finding is of clinical value to reducing fatigue, enhancing treatment adherence and therefore efficacy, and improving disease prognosis. 
A lack of studies investigating the effects of combined interventions on muscular endurance and social functioning meant complete statistical analysis could not be completed. This finding therefore warrants further research into these areas in future randomised controlled trials.

The four remaining factors showed non-significant lasting improvements following interventions consisting of both resistance and endurance exercise. This means there are overall, no statistically significant lasting effects of combined resistance and endurance interventions on physical fitness and mental wellbeing in female breast cancer patients ( $\geq 18$ years old) undergoing adjuvant therapy compared to adjuvant therapy alone, which is summarised in Table 3 in the appendix.

Despite being non-significant, these findings indicate there are still clinical benefits of combined exercise interventions to these adjuvant therapy side effects. Firstly, these findings show combined interventions elicit small improvements in cardiorespiratory fitness which is supported by other meta-analyses such as Furmaniak, Menig and Markes [38] and Lahart et al. [39] who show exercise interventions during and after adjuvant therapy non-significantly and significantly improve cardiorespiratory fitness respectively. This is reinforced by Wiestad et al. [40] and Møller et al. [41] who found exercise interventions elicit significant long-lasting improvements in cardiorespiratory fitness following adjuvant therapy. The present finding therefore implies combined exercise interventions enhance cardiorespiratory fitness which may contribute to enduring amelioration of physical fitness following adjuvant therapy. This is however modulated by ethnicity as shown by Dieli-Conwright et al. [42] who found that patients of Hispanic origin had lower baseline cardiorespiratory fitness following adjuvant treatment so would have lower overall cardiorespiratory fitness after completing combined exercise interventions compared to other ethnic groups. This suggests exercise interventions should be tailored accordingly during adjuvant therapy to maximise the lasting clinical benefits to cardiorespiratory fitness and therefore physical fitness.

Secondly, the present findings indicate there to be clinical benefits of combined interventions to muscular strength (shown by the 0.47 effect size) despite being non-significant. Support for this is provided by two recent meta-analyses conducted by Lahart et al. [39] and Møller et al. [41] who both found combined exercise interventions elicit significant enduring improvements in muscular strength following adjuvant therapy. Thus, combined exercise interventions may offer long-lasting clinical amelioration of muscular strength when completed during adjuvant treatment contributing to enhanced physical functioning and clinical outcome.

This research also shows there to be enduring clinical benefits of combined interventions to ameliorating depression demonstrated with an effect size of -0.42 . Meta-analyses by Carayol et al. [35]; Furmaniak, Menig and Markes [38] and Lahart et al. [39] support this by finding significant enduring improvements in depression in response to combined exercise interventions during adjuvant treatment. These effects may also be applied if the exercise interventions are completed after adjuvant therapy [43]-[45].

Ameliorating global fatigue, cardiorespiratory fitness, muscular strength and depression may collectively contribute to enhanced physical fitness and mental wellbeing and therefore improved QOL as demonstrated by these meta-analyses. The beneficial effects of exercise interventions on QOL are 
confirmed by additional meta-analyses such as research by Lee and Lee [46]; Carayol et al. [35]; Furmaniak, Menig and Markes [38] and Lahart et al. [39] who all found significant improvements in QOL following exercise interventions. This is also demonstrated on a singular basis by randomised controlled trials conducted by Kirkham et al. [47] and Dieli-Conwright et al. [43]. The present findings in conjunction with previous research therefore clearly show the lasting benefits of combined exercise interventions to side effects harming physical fitness and mental wellbeing during adjuvant therapy when treating breast cancer.

Depression leading to decreased QOL and mental wellbeing may arise from adjuvant therapy such as chemotherapy through a disruption in monoamine homeostasis (monoamine hypothesis). Smith [48] explains this by suggesting that since chemotherapy is non-specific during treatment, damage associated molecular patterns may arise from both tumourigenic and healthy cells. These subsequently bind to pattern recognition receptors such as Toll-like receptors (TLRs) to stimulate pro-inflammatory pathways, including NF-KB. Resulting from this, secreted pro-inflammatory cytokines such as TNF-a may increase the reuptake of several neurotransmitters including serotonin, dopamine, noradrenaline and bone-derived neurotrophic factor (BDNF) resulting in lower serum levels leading to symptoms of depression. Therefore, a mechanistic basis for these findings in improving mental wellbeing after exercise may lie in

biochemical alterations to these monoamines in response to exercise. Research by Helmich et al. [49] and Basso and Suzuki [50] show exercise induces serum increases in serotonin, dopamine, norepinephrine and BDNF [51]. Therefore, it may be postulated that serum increases in monoamine levels following exercise interventions during chemotherapy may work to restore monoamine homeostasis alleviating depressive symptoms thus improving QOL.

A mechanism for why combined exercise interventions improve muscular strength and therefore physical fitness may lie in leukocyte alterations following exercise. Generally, the role of leukocytes in muscle repair and hypertrophy is well characterised: in response to acute myotrauma, a pro-inflammatory response occurs, establishing a chemotactic gradient for leukocyte invasion. These leukocytes augment this inflammation by secreting growth factors and cytokines to stimulate satellite cell recruitment for repair [52]. Alongside satellite cells, M2 macrophages assist in repair and hypertrophy by modulating inflammation and aiding in the formation of novel myofibers and myonuclei [53]-[55]. In healthy individuals, leukocyte levels are within the normal range meaning muscle regeneration after exercise occurs normally, however chemotherapy regimens in breast cancer patients can significantly decrease blood leukocyte counts [56]. This may result in impaired muscle repair following exercise, leading to decreased muscular strength and hypertrophy after completing daily tasks during adjuvant treatment. Over time since repair is impaired, muscular strength and health may decline leading to decreased physical fitness during adjuvant treatment. This would not only account for why chemotherapy has detrimental effects on physical fitness but also why exercise interventions may improve muscular strength following adjuvant treatment. To elaborate on this, following exercise bouts, leukocyte counts significantly increase [57] which may improve muscular regeneration and hypertrophy after exercise. In addition to this, recent research shows in response to exercise, epigenetic alterations occur in leukocytes favouring the demethylation and activation of anabolic pathways such as growth hormone-releasing 
hormone improving muscular hypertrophy and regeneration [58]. Thus, the beneficial effects of exercise interventions on muscular strength may be mediated by increased leukocyte counts and alterations in the leukocyte epigenetic landscape favouring hypertrophy and repair. To complement this, exercise interventions such as endurance exercise are well characterised to improve oxygen uptake, enhancing cardiorespiratory fitness, which may in turn result in higher muscle oxygenation and therefore enhanced performance, leading to enhanced physical strength and fitness following adjuvant therapy. Holistically, improving muscular strength and health is of clinical importance to avoid the development of sarcopenia which may be augmented by adjuvant therapies, preventing the deterioration of physical fitness, QOL and mental wellbeing [59], [60].

The present findings also show interventions consisting of solely resistance exercise have an enduring, albeit non-significant, effect on improving each of the factors, apart from depression where there is little/no effect. These findings align with previous meta-analyses [61], [62]. The findings also suggest endurance interventions improve each factor excluding muscular strength in which it has a small negative impact. A rationale behind this unexpected result could be that endurance interventions elicit high levels of autophagy resulting in muscle protein breakdown exceeding synthesis leading to loss of muscle mass and strength [63]. However, the current paradigm based on an array of research suggests the opposite in that autophagy is key for muscle maintenance and homeostasis. Therefore, an alternative mechanism may be that endurance interventions induce transient muscle fiber type transitions from type II to type I fibers over the intervention period, increasing muscular endurance at the expense of muscular strength [64]. This rationale aligns with the present results.

These findings indicate that overall, resistance exercise interventions are more effective than endurance exercise to lastingly improve these adjuvant therapy side effects when performed alone. This is evident in both the separate meta-analyses and the resistance and endurance vs endurance meta-analysis in which adding resistance to endurance is more effective than endurance alone.

\subsection{Limitations}

Despite deploying methodology to minimise bias, there are still some important limitations to consider. Firstly, some of these meta-analyses are negatively impacted by studies with small sample sizes. Alongside this, multiple analyses suffer from high heterogeneity which together, may lead to low statistical power as shown by some of these analyses. This may leave these analyses prone to type 2 errors and bias leading to the possibility of misinformed conclusions. In addition, some of these metaanalyses are limited by study availability due to authors not replying with the required information and due to a lack of research in these areas. The possibility of missed papers during study selection also cannot be ruled out, although rigorous measures were taken to minimise this risk. In addition, the future direction provided by some prediction intervals were not clear, possibly impeding conclusions. These limitations therefore warrant further research into some of these adjuvant therapy factors to further inform clinical recommendations during adjuvant therapy.

\subsection{Future research}

Page 14/26 
These findings indicate that due to a lack of studies, more research is required in the following areas: the effects of combined interventions on depression, muscular endurance and social functioning, the effects of resistance interventions on cardiorespiratory fitness, depression and muscular endurance, and the effects of endurance exercise on cardiorespiratory fitness, depression, muscular endurance, muscular strength and social functioning. Additionally, due to a lack of power and non-definitive prediction intervals, further research is warranted in the following areas: the effects of combined interventions on $\mathrm{QOL}$, the effects of resistance interventions on QOL and social functioning and finally, the effects of endurance interventions on global fatigue and QOL.

In conclusion, these findings show combined exercise interventions elicit significant enduring benefits to global fatigue during adjuvant therapy. They also suggest there to be lasting clinical benefits of combined interventions to improving the remaining factors thus improving physical fitness and mental wellbeing. When performed separately, these results suggest both types of interventions are beneficial in improving physical fitness and mental wellbeing. Finally, in the event combined interventions cannot take place, interventions consisting of solely resistance exercise elicit higher clinical benefits than endurance interventions.

\section{Declarations}

Availability of data and material (data transparency): All data available from the published papers and the authors therein.

Code availability (software application or custom code): All base code can be found from the online version of "Doing Meta-Analysis With R: A Hands-On Guide". Please refer to reference 15. Examples of modified versions of the code for these analyses are available upon request.

Acknowledgements: The authors wish to thank Dr Natalie Pearson for her guidance and assistance of the principal researcher, Jonathon Mok, with this systematic review and meta-analysis.

Authors' contributions: JM and MAM designed the study; JM performed the systematic review, derived the models, and analysed the data. MJB assisted with data analysis. JM wrote the manuscript in consultation with MAM and ECA. All authors read and approved the final manuscript.

\section{Additional Information/Declarations}

Funding: This work received no external funding but was supported by Loughborough University.

Conflicts of interest: Jonathon Mok, Marie-Juliet Brown, Elizabeth C Akam and Mhairi A Morris declare that they have no conflict of interest.

\section{References}


1. Sung, H. et al. Global cancer statistics 2021: GLOBOCAN estimates of incidence and mortality worldwide for 36 cancers in 185 countries. CA. Cancer J. Clin, https://doi.org/10.3322/caac.21492 (2020).

2. Nounou, M. I. et al. Breast cancer: Conventional diagnosis and treatment modalities and recent patents and technologies supplementary issue: Targeted therapies in breast cancer treatment. Breast Cancer Basic Clin. Res, 9, 17-34 https://doi.org/10.4137/BCBCR.S29420 (2015).

3. Miller, K. D. et al. Cancer treatment and survivorship statistics, 2019. CA. Cancer J. Clin, 69 (no. 5), 363-385 https://doi.org/10.3322/caac.21565 (2019).

4. Rossi, L. et al. Impact of adjuvant chemotherapy on breast cancer survival: A real-world population. PLoS One, 10, 71-13 https://doi.org/10.1371/journal.pone.0132853 (2015).

5. Haidinger, R. \& Bauerfeind, I. Long-Term side effects of adjuvant therapy in primary breast cancer patients: Results of a web-based survey. Breast Care, 14 (no. 2), 111-116 https://doi.org/10.1159/000497233 (2019).

6. Van Moll, C. C. A., Schep, G., Vreugdenhil, A., Savelberg, H. H. C. M. \& Husson, O. The effect of training during treatment with chemotherapy on muscle strength and endurance capacity: A systematic review. Acta Oncol. (Madr), 55 (no. 5), 539-546 https://doi.org/10.3109/0284186X.2015.1127414 (2016).

7. McTiernan, A., Kooperberg, C., White, E. \& Vogel, V. G. Recreational physical activity and the risk of breast cancer in postmenopausal women: The women's health initiative cohort study. Breast Dis, 15 (no. 1), 25-26 https://doi.org/10.1097/01.ogx.0000115859.71446.89 (2004).

8. Palesh, O. et al. Physical Activity and Survival in Women with Advanced Breast Cancer. Cancer Nurs, https://doi.org/10.1097/NCC.0000000000000525 (2018).

9. Kraemer, W. J. \& Ratamess, N. A. Fundamentals of Resistance Training: Progression and Exercise Prescription. Med. Sci. Sports Exerc, 36 (no. 4), 674-688 https://doi.org/10.1249/01.MSS.0000121945.36635.61 (2004).

10. Morici, G. et al. "Endurance training: Is it bad for you?." Breathe, vol. 12, no. 2, pp. 140-147, 2016, doi: $10.1183 / 20734735.007016$.

11. Moher, D., Liberati, A., Tetzlaff, J. \& Altman, G. D. Preferred reporting items for systematic reviews and meta-analyses: The PRISMA statement. PLoS Med, 6 (no. 7), https://doi.org/10.1371/journal.pmed.1000097 (2009).

12. Document, T. "OHAT Risk of Bias Rating Tool for Human and Animal Studies Organization of This Document Indirectness, Timing, and Other Factors Related to Risk of Bias." no. January, pp. 1-37 2015.

13. Inthout, J., loannidis, J. P. \& Borm, G. F. The Hartung-Knapp-Sidik-Jonkman method for random effects meta-analysis is straightforward and considerably outperforms the standard DerSimonianLaird method. BMC Med. Res. Methodol, 14 (no. 1), 1-12 https://doi.org/10.1186/1471-2288-14-25 (2014). 
14. Panityakul, T., Bumrungsup, C. \& Knapp, G. On Estimating Residual Heterogeneity in Random-Effects Meta-Regression: A Comparative Study. J. Stat. Theory Appl, 12 (no. 3), 253 https://doi.org/10.2991/jsta.2013.12.3.4 (2013).

15. Harrer, M., Cuijpers, P., Furukawa, T. A. \& Ebert, D. D. Doing Meta-Analysis With R: A Hands-On Guide, 1st ed. Boca Raton, FL and London: Chapman \& Hall/CRC Press 2021. [Online]. Available: https://www.routledge.com/Doing-Meta-Analysis-with-R-A-Hands-On-Guide/Harrer-CuijpersFurukawa-Ebert/p/book/9780367610074

16. Higgins, J. P. T., Thompson, S. G., Deeks, J. J. \& Altman, D. G. Measuring inconsistency in metaanalyses. Br. Med. J, https://doi.org/10.1136/bmj.327.7414.557 (2003).

17. Cornette, T. et al. "Effects of home-based exercise training on VO2 in breast cancer patients under adjuvant or neoadjuvant chemotherapy (SAPA): a randomized controlled trial." Ed. MINERVA MEDICA, 2016.

18. Dong, X. et al. A longitudinal study of a multicomponent exercise intervention with remote guidance among breast cancer patients. Int. J. Environ. Res. Public Health, 17 (no. 10), 1-13 https://doi.org/10.3390/ijerph17103425 (2020).

19. Cornette, T. et al.. "Abstract P3-09-10: Adapted physical activity effect on aerobic function in patients with breast cancer treated with adjuvant or neoadjuvant chemotherapy." Cancer Res., 2013.

20. Travier, N. et al. Effects of an 18-week exercise programme started early during breast cancer treatment: A randomised controlled trial. BMC Med, 13 (no. 1), 1-11 https://doi.org/10.1186/s12916015-0362-z (2015).

21. Van Waart, H. et al. Effect of low-intensity physical activity and moderate- to high-intensity physical exercise during adjuvant chemotherapy on physical fitness, fatigue, and chemotherapy completion rates: Results of the PACES randomized clinical trial. J. Clin. Oncol, 33 (no. 17), 1918-1927 https://doi.org/10.1200/JC0.2014.59.1081 (2015).

22. Casla, S. et al. Supervised physical exercise improves V02max, quality of life, and health in early stage breast cancer patients: a randomized controlled trial. Breast Cancer Res. Treat, 153 (no. 2), 371-382 https://doi.org/10.1007/s10549-015-3541-x (2015).

23. Schmidt, T., Weisser, B., Jonat, W., Baumann, F. T. \& Mundhenke, C. Gentle strength training in rehabilitation of breast cancer patients compared to conventional therapy. Anticancer Res, 32 (no. 8), 3229-3233 (2012).

24. Husebø, A. M. L., Dyrstad, S. M., Mjaaland, I., Søreide, J. A. \& Bru, E. Effects of scheduled exercise on cancer-related fatigue in women with early breast cancer. Sci. World J, 2014, https://doi.org/10.1155/2014/271828 (2014).

25. Bolam, K. A., Mijwel, S., Rundqvist, H. \& Wengström, Y. Two-year follow-up of the OptiTrain randomised controlled exercise trial. Breast Cancer Res. Treat, 175 (no. 3), 637-648 https://doi.org/10.1007/s10549-019-05204-0 (2019).

26. Schmidt, M. E. et al. Effects of resistance exercise on fatigue and quality of life in breast cancer patients undergoing adjuvant chemotherapy: A randomized controlled trial. Int. J. Cancer, 137 (no. 2), 
471-480 https://doi.org/10.1002/ijc.29383 (2015).

27. Steindorf, K. et al.. "Randomized, controlled trial of resistance training in breast cancer patients receiving adjuvant radiotherapy: Results on cancer-related fatigue and quality of life. Ann. Oncol, 25 (no. 11), 2237-2243 https://doi.org/10.1093/annonc/mdu374 (2014).

28. Schmidt, T. et al. Comparing Endurance and Resistance Training with Standard Care during Chemotherapy for Patients with Primary Breast Cancer. Anticancer Res, 35 (no. 10), 5623-5629 (2015).

29. Cešeiko, R. et al.. "The impact of maximal strength training on quality of life among women with breast cancer undergoing treatment. Exp. Oncol, 41 (no. 2), 166-172 https://doi.org/10.32471/exponcology.2312-8852.vol-41-no-2.13249 (2019).

30. Cešeiko, R. et al.. "Heavy Resistance Training in Breast Cancer Patients Undergoing Adjuvant Therapy. Med. Sci. Sports Exerc, 52, 61239-61247 https://doi.org/10.1249/MSS.0000000000002260 (2020).

31. Wiskemann, J. et al. Effects of 12-week resistance training during radiotherapy in breast cancer patients. Scand. J. Med. Sci. Sport, 27 (no. 11), 1500-1510 https://doi.org/10.1111/sms.12777 (2017).

32. Al-Majid, S., Wilson, L. D., Rakovski, C. \& Coburn, J. W. Effects of Exercise on Biobehavioral Outcomes of Fatigue During Cancer Treatment: Results of a Feasibility Study. Biol. Res. Nurs, 17 (no. 1), 40-48 https://doi.org/10.1177/1099800414523489 (2015).

33. An, K. Y. et al.. "Effects of exercise dose and type during breast cancer chemotherapy on longer-term patient-reported outcomes and health-related fitness: A randomized controlled trial. Int. J. Cancer, 146 (no. 1), 150-160 https://doi.org/10.1002/ijc.32493 (2020).

34. Courneya, K. S. et al.. "A multicenter randomized trial of the effects of exercise dose and type on psychosocial distress in breast cancer patients undergoing chemotherapy. Cancer Epidemiol. Biomarkers Prev, 23 (no. 5), 857-864 https://doi.org/10.1158/1055-9965.EPI-13-1163 (2014).

35. Carayol, M. et al.. "Psychological effect of exercise in women with breast cancer receiving adjuvant therapy: What is the optimal dose needed? Ann. Oncol, 24 (no. 2), 291-300 https://doi.org/10.1093/annonc/mds342 (2013).

36. Kidwell, K. M. et al. Patient-reported symptoms and discontinuation of adjuvant aromatase inhibitor therapy., 120 (no. 16), 2403-2411 https://doi.org/10.1002/cncr.28756 (2014).

37. Ruddy, K. J. et al. Persistence, adherence, and toxicity with oral CMF in older women with early-stage breast cancer (Adherence companion study 60104 for CALGB 49907). Ann. Oncol, 23 (no. 12), 30753081 https://doi.org/10.1093/annonc/mds133 (2012).

38. Furmaniak, A., Menig, M. \& Markes, M. Exercise for women receiving adjuvant therapy for breast cancer. Cochrane Database Syst. Rev, no. 9, https://doi.org/10.1002/14651858.CD005001.pub3 (2016). .www.cochranelibrary.com

39. Lahart, I., Metsios, G., Nevill, A. \& Carmichael, A. Physical activity for women diagnosed with breast cancer after adjuvant therapy: A Cochrane review summary. Cochrane Database Syst. Rev, 103 (no. 
1), https://doi.org/10.1016/j.ijnurstu.2018.12.002 (2018).

40. Wiestad, T. H. et al.. "The Phys-Can observational study: Adjuvant chemotherapy is associated with a reduction whereas physical activity level before start of treatment is associated with maintenance of maximal oxygen uptake in patients with cancer.". BMC Sports Sci. Med. Rehabil, 12 (no. 1), 1-10 https://doi.org/10.1186/s13102-020-00205-9 (2020).

41. Møller, T. et al. Physical deterioration and adaptive recovery in physically inactive breast cancer patients during adjuvant chemotherapy: a randomised controlled trial. Sci. Rep, 10 (no. 1), 1-15 https://doi.org/10.1038/s41598-020-66513-9 (2020).

42. Dieli-Conwright, C. M. et al.. "Hispanic ethnicity as a moderator of the effects of aerobic and resistance exercise on physical fitness and quality-of-life in breast cancer survivors. J. Cancer Surviv, 15 (no. 1), 127-139 https://doi.org/10.1007/s11764-020-00918-3 (2021).

43. Dieli-Conwright, C. M. et al.. "Aerobic and resistance exercise improves physical fitness, bone health, and quality of life in overweight and obese breast cancer survivors: A randomized controlled trial 11 Medical and Health Sciences 1117 Public Health and Health Services. Breast Cancer Res, 20 (no. 1), 1-10 https://doi.org/10.1186/s13058-018-1051-6 (2018).

44. Patsou, E. D., Alexias, G. D., Anagnostopoulos, F. G. \& Karamouzis, M. V. Effects of physical activity on depressive symptoms during breast cancer survivorship: A meta-analysis of randomised control trials. ESMO Open, 2 (no. 5), https://doi.org/10.1136/esmoopen-2017-000271 (2017).

45. Sprod, L. K., Hayward, R., Schneider, C. M. \& Hsieh, C. C. "Three versus six months of exercise training in breast cancer survivors." vol. 121, no. 2, pp. 413-419 2011, doi: 10.1007/s10549-010-09130.Three.

46. Lee, J. \& Lee, M. G. Effects of exercise interventions on breast cancer patients during adjuvant therapy: A systematic review and meta-analysis of randomized controlled trials. Cancer Nurs, 43 (no. 2), 115-125 https://doi.org/10.1097/NCC.0000000000000682 (2020).

47. Kirkham, A. A. et al. Maintenance of fitness and quality-of-life benefits from supervised exercise offered as supportive care for breast cancer. JNCCN J. Natl. Compr. Cancer Netw, 17 (no. 6), 695702 https://doi.org/10.6004/jnccn.2018.7276 (2019).

48. Smith, H. R. Depression in cancer patients: Pathogenesis, implications and treatment (review). Oncol. Lett, 9 (no. 4), 1509-1514 https://doi.org/10.3892/ol.2015.2944 (2015).

49. Helmich, I. et al. Neurobiological alterations induced by exercise and their impact on depressive disorders [corrected]. Clin. Pract. Epidemiol. Ment. Health, 6, 115-125 https://doi.org/10.2174/1745017901006010115 (2010).

50. Basso, J. C. \& Suzuki, W. A. The Effects of Acute Exercise on Mood, Cognition, Neurophysiology, and Neurochemical Pathways: A Review. Brain Plast, 2 (no. 2), 127-152 https://doi.org/10.3233/bpl160040 (2017).

51. Heyman, E. et al.. "Intense exercise increases circulating endocannabinoid and BDNF levels in humans-Possible implications for reward and depression., 37 (no. 6), 844-851 https://doi.org/10.1016/j.psyneuen.2011.09.017 (2012). 
52. Fu, X., Wang, H. \& Hu, P. Stem cell activation in skeletal muscle regeneration. Cell. Mol. Life Sci, 72 (no. 9), 1663-1677 https://doi.org/10.1007/s00018-014-1819-5 (2015).

53. Tidball, J. G. \& Villalta, S. A. Regulatory interactions between muscle and the immune system during muscle regeneration. Am. J. Physiol. - Regul. Integr. Comp. Physiol, 298 (no. 5), https://doi.org/10.1152/ajpregu.00735.2009 (2010).

54. Pillon, N. J., Bilan, P. J., Fink, L. N. \& Klip, A. Cross-talk between skeletal muscle and immune cells: Muscle-derived mediators and metabolic implications. Am. J. Physiol. - Endocrinol. Metab, 304 (no. 5), https://doi.org/10.1152/ajpendo.00553.2012 (2013).

55. Rigamonti, E., Zordan, P., Sciorati, C., Rovere-Querini, P. \& Brunelli, S. Macrophage plasticity in skeletal muscle repair. Biomed Res. Int, 2014, https://doi.org/10.1155/2014/560629 (2014).

56. Mellios, T., Ko, H. L. \& Beuth, J. "Impact of adjuvant chemo- and radiotherapy on the cellular immune system of breast cancer patients." In Vivo (Brooklyn)., vol. 24, no. 2, pp. 227-230 2010.

57. Neves, P. R. D. S. et al. Acute effects of high- and low-intensity exercise bouts on leukocyte counts. J. Exerc. Sci. Fit, 13 (no. 1), 24-28 https://doi.org/10.1016/j.jesf.2014.11.003 (2015).

58. Denham, J., Marques, F. Z., Bruns, E. L., O’Brien, B. J. \& Charchar, F. J. Epigenetic changes in leukocytes after 8 weeks of resistance exercise training. Eur. J. Appl. Physiol, 116 (no. 6), 1245-1253 https://doi.org/10.1007/s00421-016-3382-2 (2016).

59. Adams, S. C. et al. Impact of resistance and aerobic exercise on sarcopenia and dynapenia in breast cancer patients receiving adjuvant chemotherapy: a multicenter randomized controlled trial. Breast Cancer Res. Treat, 158 (no. 3), 497-507 https://doi.org/10.1007/s10549-016-3900-2 (2016).

60. Davis, M. P. \& Panikkar, R. Sarcopenia associated with chemotherapy and targeted agents for cancer therapy. Ann. Palliat. Med, 8 (no. 1), 86-101 https://doi.org/10.21037/apm.2018.08.02 (2019).

61. Cheema, B. S., Kilbreath, S. L., Fahey, P. P., Delaney, G. P. \& Atlantis, E. Safety and efficacy of progressive resistance training in breast cancer: a systematic review and meta-analysis. Breast Cancer Res. Treat, 148 (no. 2), 249-268 https://doi.org/10.1007/s10549-014-3162-9 (2014).

62. Padilha, C. S. et al.. "Evaluation of resistance training to improve muscular strength and body composition in cancer patients undergoing neoadjuvant and adjuvant therapy: a meta-analysis. $J$. Cancer Surviv, 11 (no. 3), 339-349 https://doi.org/10.1007/s11764-016-0592-x (2017).

63. Brandt, N., Gunnarsson, T. P., Bangsbo, J. \& Pilegaard, H. Exercise and exercise training-induced increase in autophagy markers in human skeletal muscle. Physiol. Rep, 6 (no. 7), 1-12 https://doi.org/10.14814/phy2.13651 (2018).

64. Lee, S. H., Kim, B. J., Park, D. R. \& Kim, U. H. Exercise induces muscle fiber type switching via transient receptor potential melastatin 2-dependent Ca2 + signaling. J. Appl. Physiol, 124 (no. 2), 364-373 https://doi.org/10.1152/japplphysiol.00687.2017 (2018).

\section{Figures}




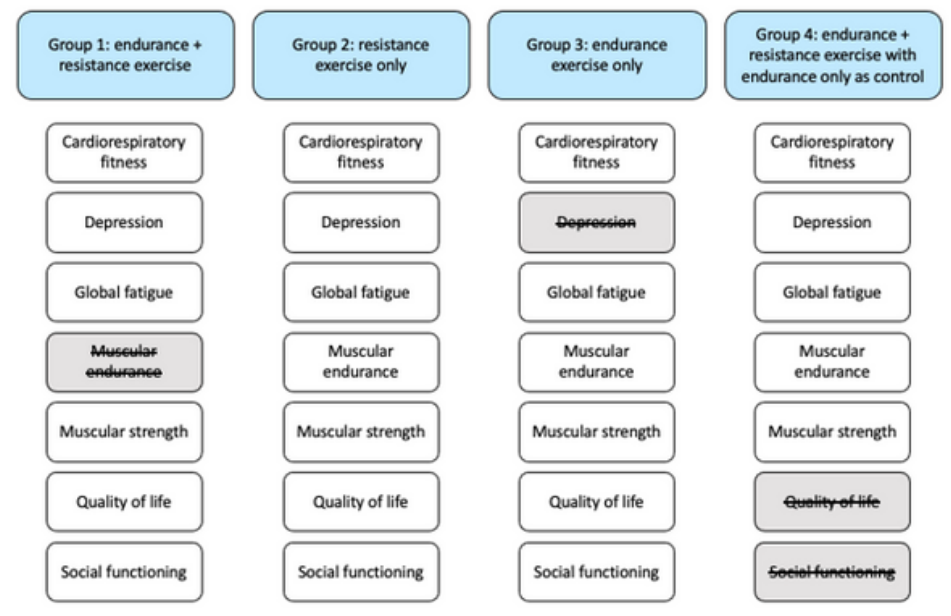

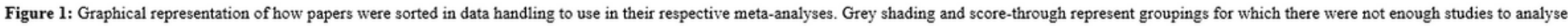
certain outcome measures.

\section{Figure 1}

\section{See image above for figure legend}
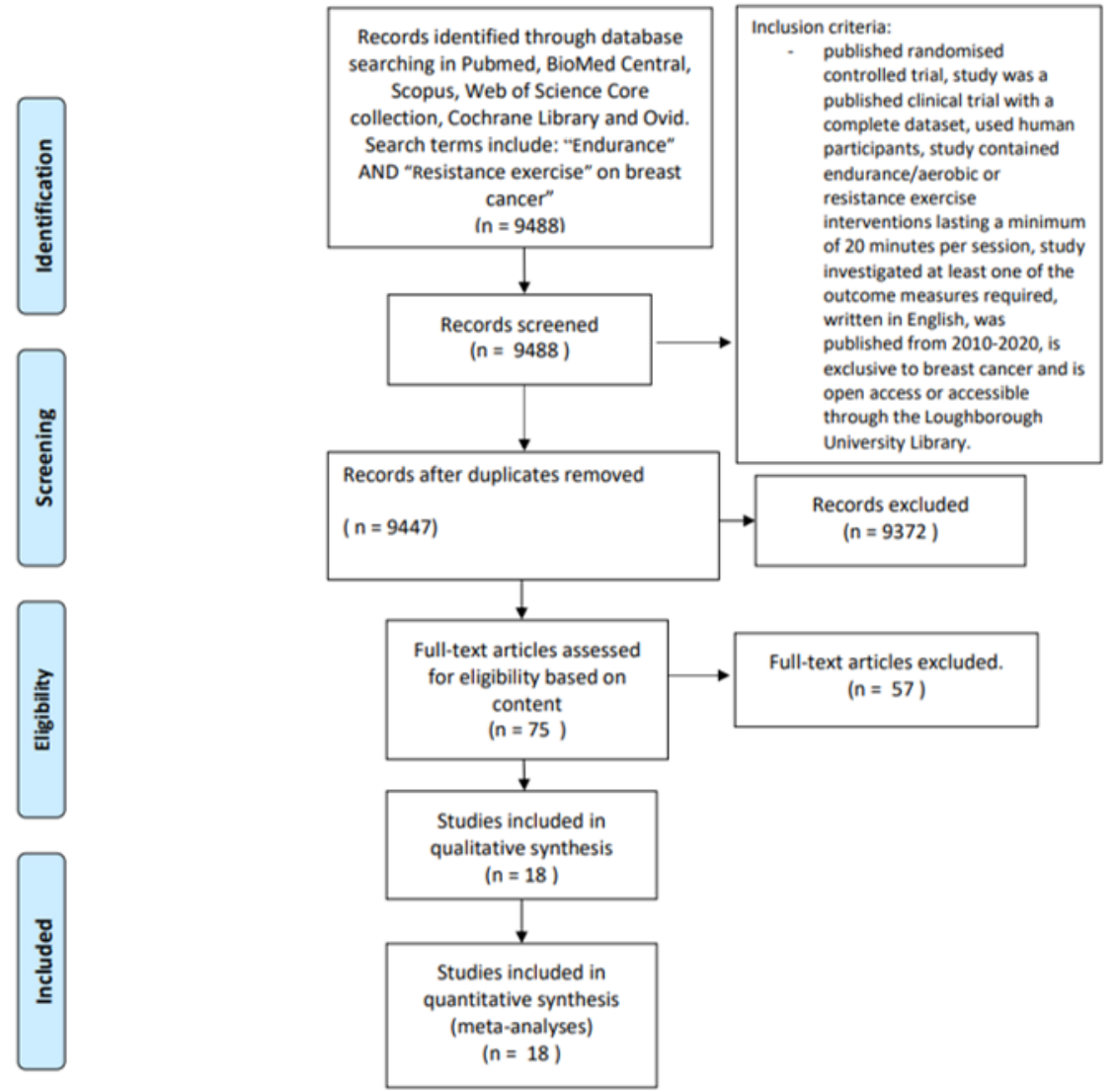

Figure 2: PRISMA 2009 flow diagram detailing the step-wise process used for study selection for these meta-analyses.

\section{Figure 2}

See image above for figure legend 


\begin{tabular}{|c|c|c|c|c|c|c|c|c|c|c|c|}
\hline Study & $\overline{\mathrm{Q} 1}$ & Q2 & $\overline{Q 3}$ & $\overline{Q 4}$ & Q5 & $\overline{Q 6}$ & $\overline{Q 7}$ & $\overline{Q 8}$ & $\overline{Q 9}$ & $\overline{Q 10}$ & Overall \\
\hline $\begin{array}{l}\text { Cornette et al. } \\
2016\end{array}$ & 4 & 4 & + & 4 & 4 & 4 & + & + & + & 4 & \\
\hline $\begin{array}{l}\text { Dong et al. } \\
2020\end{array}$ & $\uplus$ & - & $\uplus$ & $\uplus$ & - & - & 4 & 4 & $\uplus$ & + & \\
\hline $\begin{array}{l}\text { Cornette et al. } \\
2013\end{array}$ & + & + & $\oplus$ & & + & & + & + & & & \\
\hline $\begin{array}{l}\text { Travier et al. } \\
2015\end{array}$ & + & $\uplus$ & $\leftrightarrow$ & + & + & 4 & $\uplus$ & 4 & + & $\uplus$ & \\
\hline $\begin{array}{l}\text { Waart et al. } \\
2015\end{array}$ & 4 & + & + & +4 & + & + & $\uplus$ & + & 4 & 4 & \\
\hline $\begin{array}{l}\text { Casla et al. } \\
2015\end{array}$ & 4 & + & + & + & $\uplus$ & $\uplus$ & $\uplus$ & 4 & +4 & 4 & \\
\hline $\begin{array}{l}\text { Schmidt et al. } \\
2015\end{array}$ & 4 & + & 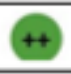 & + & $\uplus$ & 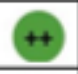 & ++ & 4 & 4 & 4 & \\
\hline $\begin{array}{l}\text { Al-Majid et al. } \\
2014\end{array}$ & + & & +4 & + & - & $\uplus$ & ++ & 4 & 4 & 4 & \\
\hline $\begin{array}{l}\text { Schmidt et al. } \\
2012\end{array}$ & $\uplus$ & $\leftrightarrow$ & + & + & & $\uplus$ & + & $\uplus$ & $\uplus$ & + & \\
\hline $\begin{array}{l}\text { Ceseiko et al. } \\
2019\end{array}$ & 4 & + & + & + & + & + & 4 & 4 & + & + & \\
\hline $\begin{array}{l}\text { Ceseiko et al. } \\
2020\end{array}$ & 4 & + & 4 & 4 & + & 4 & + & 4 & 4 & 4 & 4 \\
\hline $\begin{array}{l}\text { Steindorf et } \\
\text { al. } 2014\end{array}$ & $\uplus$ & + & 4 & + & 4 & + & + & 4 & & + & + \\
\hline $\begin{array}{l}\text { Husebs et al. } \\
2014\end{array}$ & 4 & + & 4 & 4 & + & 4 & & + & 4 & + & 4 \\
\hline $\begin{array}{l}\text { Bolam et al. } \\
2019\end{array}$ & 4 & - & 4 & 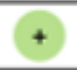 & + & + & 4 & 4 & 4 & 4 & +4 \\
\hline $\begin{array}{l}\text { Schmidt et al. } \\
2015\end{array}$ & 4 & + & + & 4 & + & + & $\uplus$ & 4 & 4 & + & \\
\hline An et al. 2020 & 4 & NR & $\oplus$ & $\uplus$ & + & & + & + & $\uplus$ & + & \\
\hline $\begin{array}{l}\text { Courneya et } \\
\text { al. } 2014\end{array}$ & $\uplus$ & $\uplus$ & $\uplus$ & $\uplus$ & + & $\uplus$ & $\uplus$ & + & + & $\uplus$ & \\
\hline $\begin{array}{l}\text { Wiskemann et } \\
\text { al. } 2017\end{array}$ & 4 & $\uplus$ & 4 & + & 4 & $\uplus$ & + & + & 4 & 4 & \\
\hline
\end{tabular}

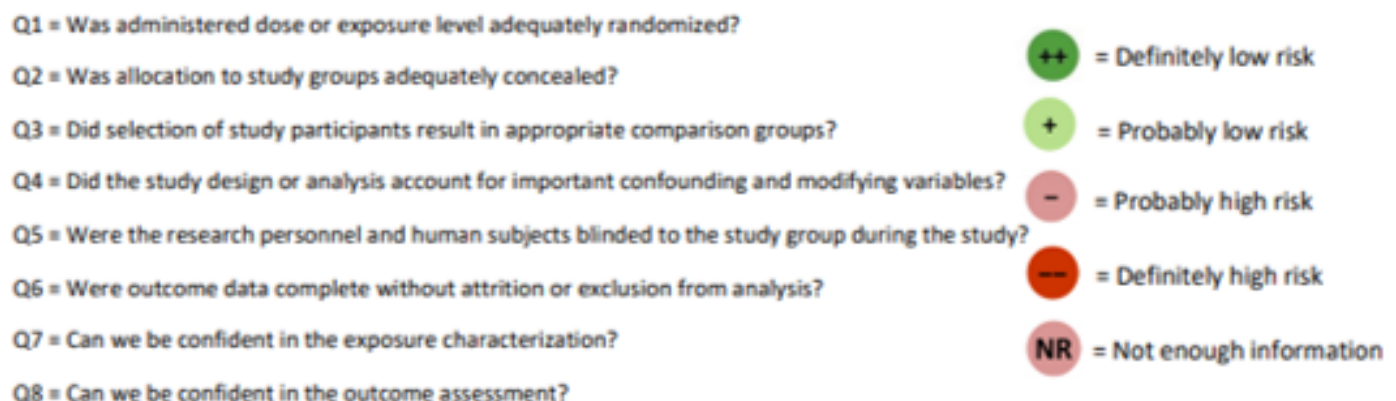

Q8 = Can we be confident in the outcome assessment?

Q9 = Were all measured outcomes reported?

Q10 = Were there no other potential threats to internal validity?

\section{Figure 3}

Risk of bias results for the 18 studies included in the meta-analyses using the OHAT rating tool. 


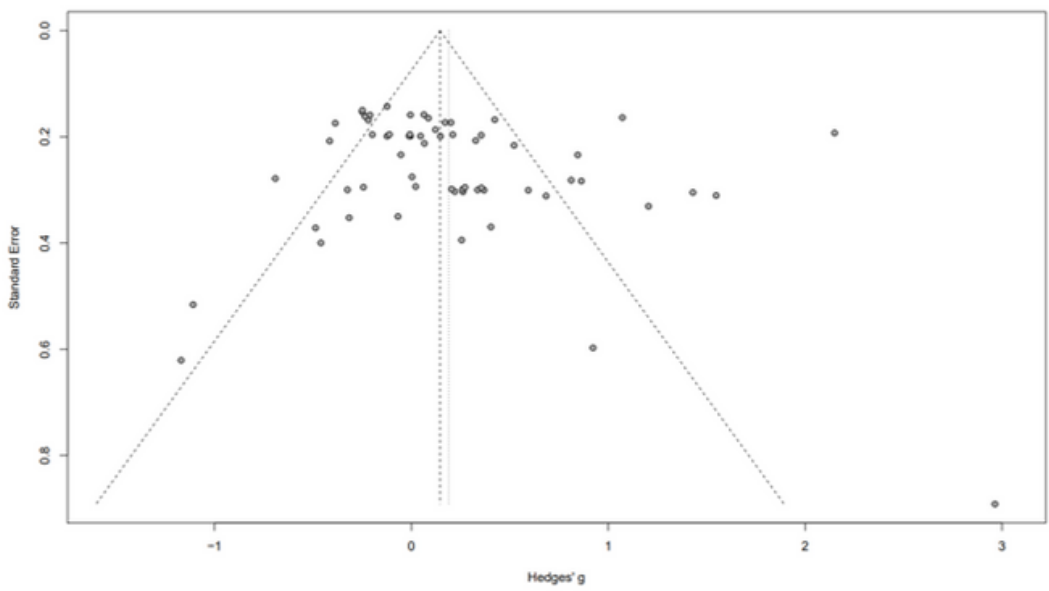

Figure 4: Funnel plot showing symmetry and therefore no publication bias in the papers used

\section{Figure 4}

See image above for figure legend 


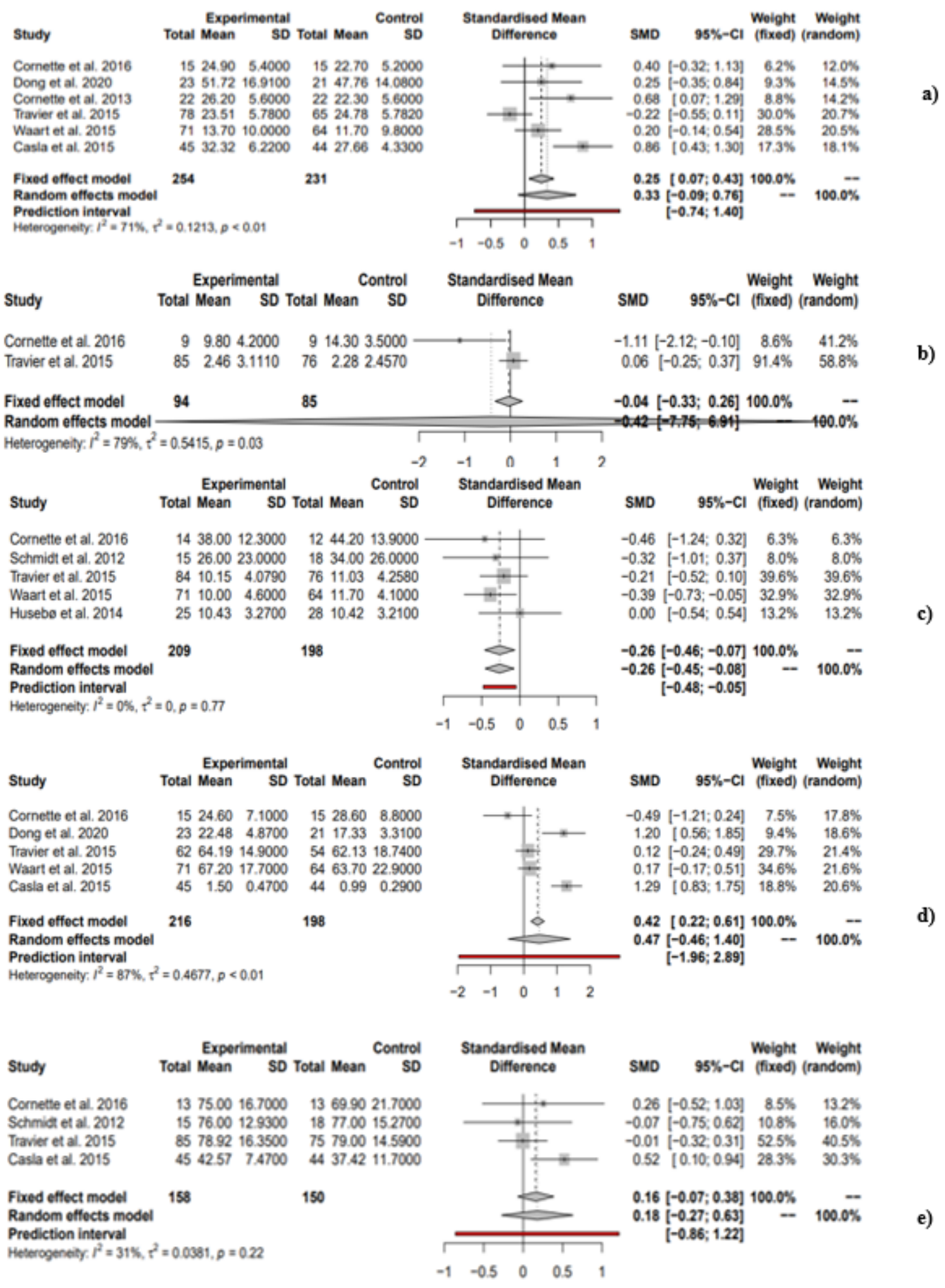

\section{Figure 5}

Random effects models showing the effects of combined resistance and endurance interventions on a) cardiorespiratory fitness, b) depression, d) muscular strength, e) quality of life during adjuvant treatment. Fixed effects model showing the effects of combined resistance and endurance interventions on c) global fatigue during adjuvant treatment. Positive effect sizes favour the exercise intervention in a), d) and e). Negative effect sizes favour the exercise intervention in b) and c). 

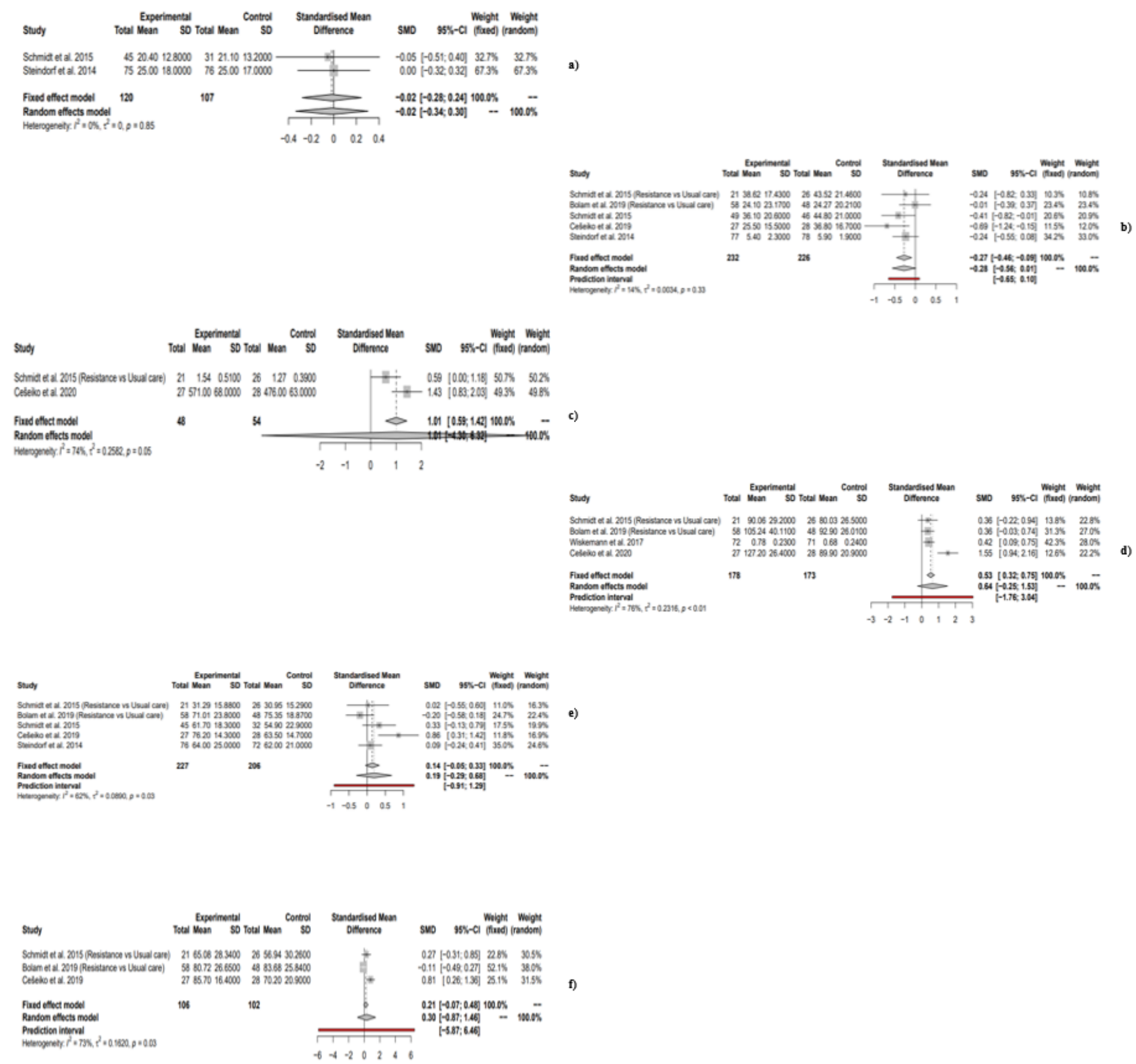

Figure 6

Random effects models showing the effects of resistance interventions on b) global fatigue, c) muscular endurance, d) muscular strength, e) quality of life, f) social functioning during adjuvant treatment. Fixed effects model showing the effects of resistance interventions on a) depression during adjuvant treatment. Negative effect sizes favour the exercise intervention in a) and b). Positive effect sizes favour the exercise intervention in c), d), e) and f). 


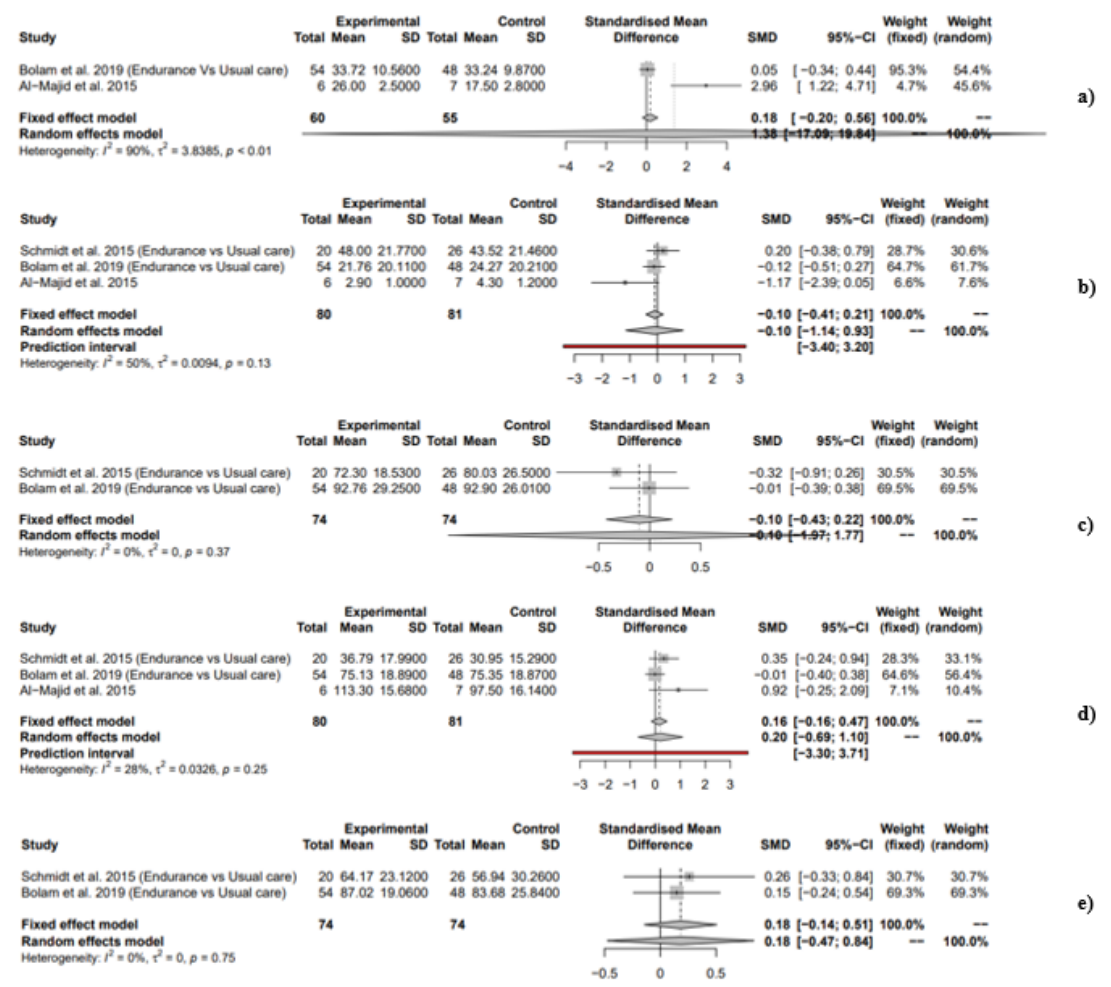

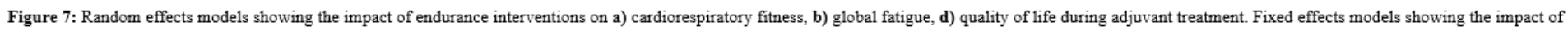
endurance interventions on c) muscular strength, e) social functioning. Positive effect sizes favour the exercise intervention in a), c), d) and e). Negative effect sizes favour the exercise intervention in b).

\section{Figure 7}

See image above for figure legend

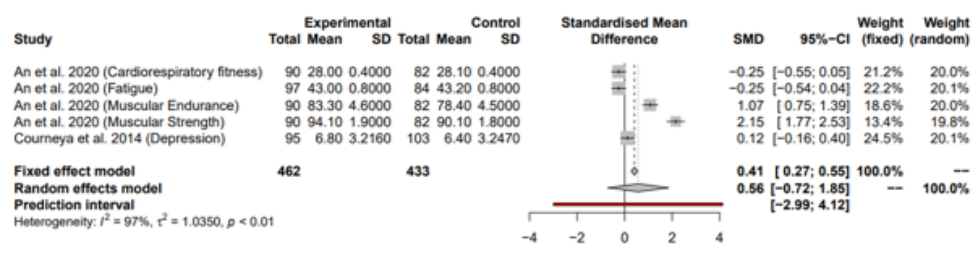

Figure 8: Random effects model showing the effects of adding resistance interventions to endurance interventions on adjuvant therapy related side effects compared to using endurance interventions alone. Positive effect sizes favour the exercise intervention.

Figure 8

See image above for figure legend

\section{Supplementary Files}

This is a list of supplementary files associated with this preprint. Click to download.

- Appendices.docx 